$\mathrm{T}$ his list celebrates the generosity of APSA members and friends in giving to one or more of the following programs from 1996 through the 2018 fiscal year: program operating support, including the Congressional Fellowship Program and the Centennial Center for Political Science and Public Affairs; initiatives promoting gender, racial and ethnic diversity, including the Fund for Latino Scholarship and Ralph Bunche Summer Institute; teaching and research fellowships; professional awards; faculty and advanced graduate student travel grants. Thank you for ensuring that the benefits of membership and influence of the profession will extend to many more generations of scholars.

The generosity of our members and friends makes us stronger. By making a gift to APSA, programming that promotes a vibrant future for the discipline is made possible. For more information on how you can make a lasting impact on APSA's work through a planned gift, or other ways that you can give, contact Jason Sapia, Development Director, at jsapia@apsanet.org. APSA updates this list annually in the January issue of $P S$.

\begin{tabular}{|c|c|}
\hline \multirow{2}{*}{$\begin{array}{l}\text { CENTENNIAL CIRCLE } \\
(\$ 25,000+)\end{array}$} & Robert Merry \\
\hline & Norman H. Nie \\
\hline Walter E. Beach & Jack W. Peltason \\
\hline Doris A. Graber & Nancy Waclawek \\
\hline Pendleton Herring & Stephen L. Wasby \\
\hline Chun-tu Hsueh & Pi Sigma Alpha \\
\hline Irving Krauss & Policy Studies Organization \\
\hline Arend Lijphart & University of Iowa \\
\hline Elinor Ostrom & William T. Grant Foundation \\
\hline \multicolumn{2}{|l|}{ Beryl A. Radin } \\
\hline Leo A. Shifrin & 2003 CIRCLE \\
\hline Frank J. Sorauf & $(\$ 10,000+)$ \\
\hline Paul A. Volcker & Roger H. Davidson \\
\hline \multirow{2}{*}{$\begin{array}{l}\text { Andrew W. Mellon } \\
\text { Foundation }\end{array}$} & Heinz Eulau \\
\hline & Ruth S. Jones \\
\hline \multirow{2}{*}{$\begin{array}{l}\text { Carnegie Corporation of New } \\
\text { York }\end{array}$} & Martha Joynt Kumar \\
\hline & Dale Rogers Marshall \\
\hline $\begin{array}{l}\text { Japan-United States } \\
\text { Friendship Commission }\end{array}$ & Dianne M. Pinderhughes \\
\hline \multirow{2}{*}{$\begin{array}{l}\text { John D. and Catherine T. } \\
\text { MacArthur Foundation }\end{array}$} & Robert D. Putnam \\
\hline & Ronald J. Schmidt, Sr. \\
\hline National Science Foundation & Janet D. Steiger \\
\hline Russel Sage Foundation & Kenton W. Worcester \\
\hline \multirow[t]{2}{*}{ University of Louisville } & Kettering Foundation \\
\hline & Northwestern University \\
\hline $\begin{array}{l}\mathbf{1 9 0 3} \text { CIRCLE } \\
(\$ 15,000+)\end{array}$ & Smith College \\
\hline Richard F. Fenno & FOUNDER'S CIRCLE \\
\hline Jo Freeman & $(\$ 5,000+)$ \\
\hline Robert O. Keohane & Tony Affigne \\
\hline Ken Meier & \\
\hline
\end{tabular}

Barbara B. Bardes

Lucius J. Barker

Robert H. Bates

Susan C. Bourque

David W. Brady

John G. Bretting

Thomas M. Carsey

Robert F. Durant

Luis Ricardo Fraga

James L. Gibson

Betty Glad

Ira Goldstein

John Mark Hansen

John P. Harrod

Martin O. Heisler

Jennifer L. Hochschild

Robert A. Horn

Karen M. Hult

Samuel P. Huntington

Gary C. Jacobson

Malcolm E. Jewell

Bryan D. Jones

Joyce K. Kallgren

Mary Fainsod Katzenstein

Ira Katznelson

Margaret Levi

Sydnee Guyer Lipset

Theodore J. Lowi

Jane Mansbridge

Valerie J. Martinez-Ebers

Paula D. McClain

Cynthia McClintock

Ruth P. Morgan

Norman J. Ornstein

T.J. Pempel

Jewel L. Prestage

Lucian W. Pye

J. Austin Ranney

Ben F. Reeves

Paul J. Rich

David B. Robertson

Catherine E. Rudder

Kay Lehman Schlozman

Eric J. Scott
J. Merrill Shanks

Lee Sigelman

Howard J. Silver

Erica S. Simmons

William O. Slayman

Paul M. Sniderman

Jonathan R. Strand

Mary E. Stuckey

James A. Thurber

Charles V. Walker

Kenneth N. Waltz

Susan Welch

James Q. Wilson

Jason H. Windett

Richard F. Winters

Thomas R. Wolanin

Nancy H. Zingale

University of New Mexico

Yale University

\section{BUILDER'S CIRCLE}

(\$2,500+)

Alan I. Abramowitz

Martha A. Ackelsberg

David Adamany

Gabriel A. Almond

Eugene J. Alpert

James E. Anderson

Gary J. Andres

Patricia Artinian

Manuel Avalos

William I. Bacchus

Michael A. Baer

Paul Allen Beck

John F. Bibby

Amy B. Bridges

Michael A. Brintnall

David S. Broder

Charles S. Bullock III

Margaret Cawley

Philip E. Converse

William J. Daniels

Christopher J. Deering

Lawrence C. Dodd

Jorge I. Dominguez 


\begin{tabular}{|c|c|c|c|}
\hline Marion E. Doro & Julie L. Novkov & University of California, Los & Elizabeth Capell \\
\hline Melvin J. Dubnick & Karen O'Connor & Angeles & Kaisheka Juree Capers \\
\hline Leon D. Epstein & David Scott Palmer & $\begin{array}{l}\text { University of North Carolina, } \\
\text { Chapel Hill }\end{array}$ & K. Juree Capers \\
\hline Kathleen A. Frankovic & Leonard Parkinson & University of Oslo & Lief H. Carter \\
\hline Beth C. Fuchs & Carole Pateman & University of Texas, Austin & Gretchen G. Casper \\
\hline John Armando Garcia & Desiree S. Pedescleaux & Walnut Foundation & Bruce E. Caswell \\
\hline George J. Graham & Gerald M. Pomper & Women's Caucus for Political & Leonard Champney \\
\hline Virginia H. Gray & Mary Cornelia Porter & Science & Daniel S. Cheever \\
\hline Joel B. Grossman & James J. Prestage & & Vice President Richard \\
\hline Charles D. Hadley & George H. Quester & SECOND CENTURY & Cheney \\
\hline Glen A. Halva-Neubauer & Nicol C. Rae & $\begin{array}{l}\text { SOCIETY } \\
(\$ 1,000+)\end{array}$ & Beverly A Cigler \\
\hline Robert J-P. Hauck & Randall B. Ripley & Gabriele Abels & Clarke E. Cochran \\
\hline Mary Hawkesworth & Ronald L. Rogowski & John H. Aldrich & Louise K. Comfort \\
\hline Jeffrey R. Henig & Alan Rosenthal & Herbert E. Alexander & Sara Connor \\
\hline Mary A. Hepburn & Mildred A. Schwartz & Hayward R. Alker & M. Margaret Conway \\
\hline John F. Hoadley & Gary M. Segura & Herbert B. Asher & Martha Crenshaw \\
\hline Anne H. Hopkins & Kenneth Sherrill & David Austen-Smith & Thomas E. Cronin \\
\hline Robert Huckfeldt & W. Phillips Shively & Robert Axelrod & Milton C. Cummings, Jr. \\
\hline Vincent L. Hutchings & Roberta S. Sigel & Seth B. B. Benjamin & Robert A. Dahl \\
\hline Patricia W. Ingraham & Barbara Sinclair & Judith A. Baer & Vincent Davis \\
\hline Kent Jennings & Steven Rathgeb Smith & Lee Ann Banaszak & Michael C. Dawson \\
\hline Robert Jervis & John D. Sprague & Maryann Barakso & Richard E. DeLeon \\
\hline Loch K. Johnson & Harvey Starr & Samuel H. Barnes & Patrick J. Deneen \\
\hline Charles O. Jones & Judith H. Stiehm & Charles J. Barrilleaux & Louis DeSipio \\
\hline Matthew Holden, Jr. & James A. Stimson & Larry M. Bartels & Alexander A. Doska \\
\hline Joseph Stewart, Jr. & Camilla M. Stivers & Harold F. Bass & Delmer D. Dunn \\
\hline William J. Keefe & Clarence N. Stone & Pamela Bataillon & Thomas K. Dye \\
\hline Charles E. King & Sidney Tarrow & Lewis Bateman & George C. Luwdras int \\
\hline Herbert Kitschelt & Sue Tolleson-Rinehart & Frank R. Baumgartner & Cynthia Enloe \\
\hline Allan Kornberg & Toni-Michelle Travis & Lisa Garcia Bedolla & Maria J. Falc \\
\hline Kay Lawson & David B. Truman & Samuel H. Beer & Victoria A. Farrar-Mye \\
\hline Avery Leiserson & Sidney Verba & Jeffrey M. Berry & Michael K. Fauntroy \\
\hline Burdett A. Loomis & David Vogel & Henry S. Bienen & Martha Finnemore \\
\hline Susan A. MacManus & John C. Wahlke & James Warner Bjorkman & Morris Fiorina \\
\hline David B. Magleby & Charles E. Walcott & Christopher Bosso & James S. Fishkin \\
\hline Thomas E. Mann & Charles E. Walker & Ann O'M. Bowman & Lynne E. Ford \\
\hline David R. Mares & Brandon Walton & Janet M. Box-Steffensmeier & Jane E. Fountain \\
\hline Joel Margolis & Paul J. Weber & Richard W Bovd & Linda L. Fowler \\
\hline David R. Mayhew & S. Laurel Weldon & John Brademas & George Frederickson \\
\hline Daniel A. Mazmanian & Graham K. Wilson & JणIII Diactias & William E. Frenzel \\
\hline Dorothy E. McBride & Lois R. Wise & Steven I. Brams & Jerry Warden Friedheim \\
\hline Eileen McDonagh & Maurice C. Woodard & 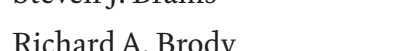 & Jurg Martin Gabriel \\
\hline Lorraine M. McDonnell & Eastern Michigan University & Mitchell Brown & F. Chris Garcia. \\
\hline Nancy R. McWilliams & Harold D. Lasswell Society & 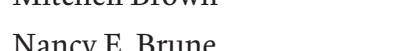 & Louis C. Gawthrop \\
\hline Melissa R. Michelson & H.D. Smith Foundation & Frances Burke & Irwin N. Gertzog \\
\hline Jeffrey R. Miller & Juan March Institute & James MacGreoor Bu & Judith Lynn Goldstein \\
\hline Christopher Z. Mooney & Missouri Political Science & Nancy Burns & Samuel K. Gove \\
\hline James A. Morone & & & Fred I. Greenstein \\
\hline
\end{tabular}


Susan Webb Hammond

Fredrick C. Harris

Roderick P. Hart

Kerry L. Haynie

Lenneal J. Henderson

C. Randall Henning

Rodney E. Hero

Paul S. Herrnson

Donald V. Hester

Milton E. Hill

Kim Quaile Hill

Ole R. Holsti

J. Woodford Howard, Jr.

Evelyne Huber

Robert J. Huckshorn

William E. Hudson

Robert P. Huefner

Dennis S. Ippolito

John Ishiyama

Kenneth Janda

James Jennings

Bruce W. Jentleson

Calvin C. Jillson

Nolan E. Jones

Mark P. Jones

Michael Jones-Correa

Stanley Kelley, Jr.

Jane Y. Junn

Melvin A. Kahn

Nancy Kassop

Rita Mae Kelly

Robin A. Kolodny

Samuel Krislov

Michael C. Ladam

Janet M. Laible

Thomas P. Lauth

David L. Leal

Frances E. Lee

David C. Leege

Gregory B. Lewis

Seymour Martin Lipset

Gerhard Loewenberg

Linda Lopez

Nancy Sue Love

DeWayne L. Lucas

Arthur Lupia

Cherie Maestas

L. Sandy Maisel
Melanie Frances Manion

Sheilah Mann

Benjamin Marquez

Janet M. Martin

Karen M. McCurdy

Richard L. Merritt

Warren E. Miller

Alison Rios Millett McCartney

William Mishler

Charles L. Mitchell

Robert Montjoy

Lois B. Moreland

Richard G. Niemi

Helmut Norpoth

John S. Odell

Marion Orr

Kenneth T. Palmer

Thomas L. Pangle

Glenn R. Parker

William D. Pederson

Mark A. Peterson

John J. Pitney, Jr.

Nelson W. Polsby

Lynda W. Powell

Henry J. Pratt

Barbara A. Presnall

Michael B. Preston

Kenneth Prewitt

David E. Price

Philip L. Pritchett

Adam Przeworski

Rosemary A. Putnam

Lyn Ragsdale

Andree E. Reeves

Thomas F. Remington

Chester D. Rhoan

Leroy N. Rieselbach

Bert A. Rockman

Richard Rose

Loren D. Ross

Donald Rothchild

Eugene Y. S. Chang

Larry J. Sabato

Scott D. Sagan

Anna Sampaio

Mark Q. Sawyer

Philippe C. Schmitter

Thomas S. Schrock
Jean Reith Schroedel

Peregrine Schwartz-Shea

Joanna Vecchiarelli Scott

Antoinette G. Sebastian

Sally Coleman Selden

Ronald G. Shaiko

Donna E. Shalala

Christine Marie Sierra

Dick Simpson

Theda Skocpol

Anne-Marie Slaughter

Elliot E. Slotnick

Duncan Snidal

Robert J. Spitzer

Thomas A. Spragens, Jr.

Harold W. Stanley

Christine Di Stefano

Robert M. Stein

Susan C. Stokes

Sybil L. Stokes

Gerald H. Stollman

Deborah Stone

Floyd E. Stoner

Dara Z. Strolovitch

Michael W. Suleiman

James L. Sundquist

Raymond Tatalovich

Elizabeth Theiss-Morse

Dennis F. Thompson

Alvin B. Tillery, Jr.

Jim J Tozzi

Joan C. Tronto

George Tsebelis

Arturo Vega

Kenneth Vogel

Craig Volden

Kenneth D. Wald

Robert S. Wallerstein

Richard D. Warden

Lionel Washington

Kenneth Weakley

Herbert F. Weisberg

Jennifer Widner

Clyde Wilcox

Melissa S. Williams

David M. Wilson

Raymond E. Wolfinger

Robert C. Wood
Elisabeth Jean Wood

Deil S. Wright

Dali L. Yang

Martynas A. Ycas

Charles E. Young

James Sterling Young

James V. Young

Robert L. Youngblood

John R. Zaller

Pamela A. Zeiser

Joseph F. Zimmerman

Elizabeth A Zitrin

Michael P. Zuckert

American Review of Public Administration

Boston University

California Community Foundation

Cambridge University Press

The Claremont Institute

Consortium For Qualitative Research

Eventbrite

The Haworth Press Inc.

University of Houston

University of Illinois at Chicago

University of New Orleans

University of Wisconsin, Milwaukee

Women's Caucus for Political Science: West

\section{ANNIVERSARY SOCIETY}

$(\$ 500+)$

Joel D. Aberbach

Janet Lee Abrahm

Yvette M. Alex-Assensoh

Stephen Amberg

Kristi Andersen

Christopher J. Anderson

John W. Anderson

Lisa Anderson

Lisa Baldez

Lawrence Baum

Christina Elizabeth Bejarano

Jonathan Benjamin-Alvarado

John C. Berg

Frances Stokes Berry

Gayle Binion 
Marc Blecher

Janet K. Boles

Henry J. Bowers

M. Kenneth Bowler

Karlyn Bowman

James M. Brasfield

Stanley C. Brubaker

Jeremy Buchman

Carol Buckland

Emmett H. Buell, Jr.

Bruce J. Bueno de Mesquita

Gerard Patrick Bushell

Michael P. Canning

David A. Caputo

Jason P. Casellas

Janet M. Clark

Susan E. Clarke

David L. Coleman

David Collier

William F. Connelly, Jr.

Joseph Cooper

Gary W. Cox

Deborah K Cupp

Charles Jeremy Curtoys

Nancy Davidson

George Tinsley Davies

Rodolfo O. de la Garza

Michelle D. Deardorff

Linda C. Degutis

Brendan J. Doherty

Delia E. Donatelli

Murray P. Dry

Georgia Duerst-Lahti

Murray Steven Edelmann

Peter Enns

Kenneth Paul Erickson

Malcolm M. Feeley

James E. Fleming

Christopher H. Foreman, Jr.

Jeffry A. Frieden

Kent Gardner

Michael Garrety

John B. Gates

Harvey Glickman

Miriam A. Golden

Marissa Martino Golden

Kenneth Goldstein

Robert T. Golembiewski
Leonard E. Goodall

Jane A. Gordon

Charles W. Gossett

Robert C. Grady

Jose Angel Gutierrez

Henry E. Hale

Melinda Gann Hall

Richard L. Hall

Susan B. Hansen

Carol Hardy-Fanta

William A. Hazleton

Virginia S. Herring

Marjorie R. Hershey

John R. Hibbing

Kim Hildred

John W. Holcombe

Xiaobo $\mathrm{Hu}$

Juan Carlos Huerta

Patricia A. Hurley

Wallace E. Hutton

Edward Thompson, III

Tomoaki Iwai

Lawrence R. Jacobs

Harold K. Jacobson

Amaney Jamal

Tobe Johnson

Richard Johnston

W. Landis Jones

Peter H. Juviler

Miles Kahler

Max M. Kampelman

Karen M. Kaufmann

Margaret E. Keck

Sean Q Kelly

Mark A. Kessler

Donald F. Kettl

Anthony King

Tyson D. King-Meadows

Samuel A. Kirkpatrick

Gary Klass

Cynthia N. Ladd

David A. Lake

Peter Lange

Bruce A. Larson

Miriam J. Laugesen

Logan A. Lee

Jan E. Leighley

Ralph Lerner
William F. Levantrosser

Mark I. Lichbach

Evan S. Lieberman

Pei-te Lien

Serge E. Logan

Robert Lorish

David Lowery

Naomi B. Lynn

Stephen Macedo

Catherine A. Mackinnon

Linda Kay Mancillas

Lynn Mather

Susan McAndrew

Rachel M. McCleary

Gwyneth McClendon

Scott D. McClurg

Nancy E. McGlen

Roger A. McGuire

Susan McWilliams

Tali Mendelberg

Carol A. Mershon

Cheryl M. Miller

H. Brinton Milward

Gwendolyn Mink

Dinshaw Mistry

Devra Coren Moehler

James Edward Monogan III

Jacob M. Montgomery

J. Donald Moon

Roy Morey

Michael E. Morrell

Robert J. Mundt

Walter F. Murphy

Richard W. Murray

Roger B Myerson

Candice J. Nelson

Dale A. Neuman

Richard E. Neustadt

Melissa Nobles

Irfan Nooruddin

Pippa Norris

Joseph S. Nye, Jr.

Rosemary O'Leary

Bruce I. Oppenheimer

Diana M. Owen

Robert A. Packenham

Dorothy A. Palmer

Anne M. Parsons
Louis Pauly

Francisco I. Pedraza

Paul C. Peterson

Mark P. Petracca

Susan J. Pharr

John R. Phillips

Anne R. Pierce

Eric Plutzer

Philip H. Pollock III

Jay W. Prestage

Melynda J. Price

Diane-Michele Prindeville

Wilbur C. Rich

Erin E. Richards

Donald L. Robinson

Pearl T. Robinson

John A. Rohr

Mark Carl Rom

Barbara S. Romzek

Raymond K. Rossiter

Susanne Hoeber Rudolph

Lloyd I. Rudolph

Barry S. Rundquist

David R. Salazar

Stephen G. Salkever

Virginia Sapiro

Elizabeth Nathan Saunders

Wendy J. Schiller

Gordon Schochet

David A. Schultz

Mary L. (Molly) Shanley

Robert Y. Shapiro

Colleen J. Shogan

Beth A. Simmons

Andrea Y. Simpson

Valeria Sinclair-Chapman

Nicholas Rush Smith

Steven S. Smith

Raphael J. Sonenshein

Adaljiza Sosa-Riddell

Robert T. Starks

R. Vladimir Steffel

Alfred C. Stepan

Laura Stoker

Randall W. Strahan

Kaare Wallace Strom

James H. Svara

John Thomas Tambornino 


\begin{tabular}{|c|c|c|c|}
\hline Sidney George Tarrow & Nancy Bermeo & Patrick J. Egan & Robert A. Heineman \\
\hline C. Neal Tate & Naomi Black & Jennifer Einspahr & Andrew P. Helene \\
\hline Kathryn Dunn Tenpas & Jeanne C. Blamey & Peter Eisinger & Charles P. Henry \\
\hline Jane Thery & Janice L. Bockmeyer & Colin Elman & Margaret G. Hermann \\
\hline Cameron G. Thies & Frederick J. Boehmke & Lee Epstein & Allen D. Hertzke \\
\hline Norman C. Thomas & Elizabeth T. Boris & Justin E. Esarey & Peter T. Higgins \\
\hline J. Ann Tickner & Mary Anne Borrelli & Amitai Etzioni & Ronald Salem Hikel \\
\hline Jeffrey K. Tulis & Eileen Hunt Botting & Timothy J Feddersen & Erik P. Hoffmann \\
\hline Ashutosh Varshney & Katya D. Bowers & Frank Feigert & Raymond F. Hopkins \\
\hline Jennifer Nicoll Victor & Patrick T. Brandt & L. Boyd Finch & Daniel J. Hopkins \\
\hline Barbara A. Vobejda & Tehama Lopez Bunyasi & Jane Flax & Robert F. Hopper \\
\hline Sherri L. Wallace & Donald J. Calista & Daniel B. Fleming & Janice H. Hopper \\
\hline Carol S. Weissert & Thomas M. Callaghy & Frederic J. Fleron, Jr. & Serge Hurtig \\
\hline Ralph R. Widner & Randall L. Calvert & Donald L. Fowler, Sr. & Holly Jarman \\
\hline David Wilsford & James E. Campbell & Jonathan A. Fox & Ted G. Jelen \\
\hline Rick K. Wilson & David T. Canon & Mark N. Franklin & Edward T. Jennings \\
\hline Frederick M. Wirt & Jason Caro & Lorrie Frasure-Yokley & E. Terrence Jones \\
\hline Tom K. Wong & Susan J. Carroll & Alisa Hicklin Fryar & Eric Gonzalez Juenke \\
\hline Kenneth K. Wong & Karl H. Cerny & Paul Frymer & Marion R. Just. \\
\hline \multirow{2}{*}{$\begin{array}{l}\text { Center for the Study of } \\
\text { Federalism }\end{array}$} & Roland Chi & Fritz Gaenslen & Kenneth Kato \\
\hline & Anne Cizmar & Brian J. Gaines & Peter J. Katzenstein \\
\hline MacArthur Foundation & John A. Clark & William A. Galston & Kate M. Kaufer \\
\hline \multirow{2}{*}{$\begin{array}{l}\text { Research4Impact,Inc. } \\
\text { Scholars Strategy Network }\end{array}$} & Rosalee A. Clawson & John G. Geer & William R. Keech \\
\hline & Myles L. Clowers & Shirley M. Geiger & Scott Keeter \\
\hline Temple University Press & Celia F. Cohen & John P. Geis II & Catherine McArdle Kelleher \\
\hline \multirow{4}{*}{$\begin{array}{l}\text { University of Chicago } \\
\text { University of Notre Dame } \\
\text { Women's Caucus for Political } \\
\text { Science: South }\end{array}$} & David Cohen & Joyce Gelb & Rogan Kersh \\
\hline & Christopher Colmo & Michael A. Genovese & Mark Kesselman \\
\hline & Constance E. Cook & Carl Gershman & Frank Kessler \\
\hline & Timothy E. Cook & John Gibbons & John Kincaid \\
\hline \multirow{2}{*}{$\begin{array}{l}\text { FRIENDS } \\
(\$ 250+)\end{array}$} & William Corlett & Clare Ginger & Justin Kirkland \\
\hline & David Covin & Marianne Githens & Thad Kousser \\
\hline Robert B. Alexander & Cary R. Covington & Esther S. Goldstein & Mary Kroeger \\
\hline J. Theodore Anagnoson & Michael C. Craw & Jeff Goodwin & Mona Lena Krook \\
\hline Michael Lee Andrews & Marian L. Currinder & George J. Gordon & Michael G. Krukones. \\
\hline Kevin Avruch & James M. Curry & Amy D. Gossett & Bruce C. Ladd, Jr. \\
\hline Q. Whitfield Ayres & Russell J. Dalton & Christina M. Greer & Steve Lagerfeld \\
\hline Lawrie Balfour & Janna L. Deitz & Bruce N. Gregory & David D. Laitin \\
\hline Donald G. Balmer & Linda deLeon & Christian R. Grose & Robert E. Lane \\
\hline Kevin K. Banda & Martha Derthick & Ashley C. Grosse & Jessica L. Lavariega Monforti \\
\hline William T. Barndt & I. M. (Mac) Destler & Kaaryn Gustafson & Kathryn C. Lavelle \\
\hline Matt A. Barreto & Patrice L. Dickerson & Clifford P. Hackett & Geoffrey C. Layman \\
\hline Edwina Barvosa & Lisa J. Disch & Peter A. Hall & Patrick Leblond \\
\hline Jackie Beilhart & Harun Dogo & Welling Hall & Wei-chin Lee \\
\hline Lauren Cohen Bell & Julie Dolan & Samuel Halperin & Howard H. Lentner \\
\hline Cristina Beltran & Michael W. Doyle & John W. Harbeson & David M. Levitan \\
\hline Seyla Benhabib & Larry Le Duc & Mai Omer Hassan & Jack S. Levy \\
\hline Elizabeth A. Bennion & Mary H. Durfee & Virginia Haufler & Jacob T. Levy \\
\hline Stanley P. Berard & Carter Edgeworth & Ken Heath & Ann Chih Lin \\
\hline
\end{tabular}




\begin{tabular}{|c|c|c|c|}
\hline Alice M. Litwinowicz & Benjamin I. Page & George C. Shipley & Bang-Soon L. Yoon \\
\hline Cary E. Losson & Robert A. Pastor & Robert C. Smith & M. Crawford Young \\
\hline George I. Lovell & Kelly D. Patterson & W. Rand Smith & Eleanor E. Zeff \\
\hline Robert C. Lowry & Bradley H. Patterson & Edmond C. Smith, Jr. & J. Nicholas Ziegler \\
\hline Sue Ellen M. Charlton & Steven A. Peterson & Etel L. Solingen & Tamas D. Zsitvay \\
\hline Dr Joseph MacKay & M. Anne Pitcher & Lester Kenyatta Spence & Brigham Young University \\
\hline Fiona S. Mackay & Paul L. Posner & Arthur Spirling & New England Political Science \\
\hline Forrest Maltzman & G. Bingham Powell, Jr. & William C. Spragens & Association \\
\hline Suzanne M. Marilley & Mahendra Prasad & Robert H. Stern & University of Baltimore \\
\hline Fletcher McClellan & Frank Prindle & Christopher T. Stout & \\
\hline Stephen D. McDowell & Doris Marie Provine & Scott Straus & $\begin{array}{l}\text { ASSOCIATES } \\
(\$ 100+)\end{array}$ \\
\hline Andrew S. McFarland & Sherman Crewell Puckett & Gregory Strizek & Rahma Abdulkadir \\
\hline Eric McGhee & Donald P. Racheter & Eileen M. Sullivan-Marx & David B. Abernethy \\
\hline Wilson Carey McWilliams & David Rayside & Elizabeth Super & Henry J. Abraham \\
\hline Nancy McWilliams & William G. Resh & Carl D. Sutton & Paul M. Abrahm \\
\hline Eugene Meehan & Jean C. Robinson & Carol M. Swain & Marisa Abrajano \\
\hline Melissa Buis Michaux & Susan Carol Rogers & Duane H. Swank & Paul R. Abramson \\
\hline Robert Mickey & Rodolfo Rosales & Alec A. Sweet & Brooke A. Ackerly \\
\hline Toni P. Miles & Guillermo Rosas & Henrik Syse & Natalie Adona \\
\hline Harris Miller & Roger P. Rose & Ross B. Talbot & Carl D. Adrianopoli \\
\hline Bruce Miroff & Laura Roselle & Jennifer Morrison Taw & Shariff Ahmad \\
\hline Sara McLaughlin Mitchell & Nancy L. Rosenblum & Dawn L. Teele & Takeshi Akiba \\
\hline Patrice Mitchell & Frances McCall Rosenbluth & Morton J. Tenzer & Oluwaseyi Sherifat Akinleye \\
\hline Heather Silber Mohamed & Jo Ellen Ross & Gwynn Thomas & Roberto Alejandro \\
\hline James P. Monroe & John E. Rouse & Santa Algeo Traugott & Nikol G. Alexander-Floyd \\
\hline Kristen Renwick Monroe & Herbert J. Rubin & Peter Trubowitz & John R. Alford \\
\hline Vincent G. Moscardelli & Andrew C. Rudalevige & Gary Tschoepe & Scott Allard \\
\hline Wynne Walker Moskop & Bruce M. Russett & Rick Valelly & Danielle Allen \\
\hline Elizabeth F. Moulds & Andrew Sabl & Paul P. Van Riper & Gar Alperovitz \\
\hline Esther N. Mwangi & Kira Sanbonmatsu & Susanne E. Vandenbosch & Mohammed Alsaud \\
\hline Peter N. Skerry, Jr. & Eve Sandberg & George Vernardakis & James E. Alt \\
\hline Katherine C. Naff & Sylvia G. Sanders & Thomas Vocino & Sharon Z. Alter \\
\hline Hitoshi Namikawa & Courtney Sanders & Mark C. Walker & Jessica Racine Altif \\
\hline Eric J. Narcisse & Christopher Michael Sands & Shirley Anne Warshaw & John A. Altman \\
\hline Carol R. Nechemias & Marjorie Sarbaugh-Thompson & Gregory R. Weiher & Winifred L. Amaturo \\
\hline Jennifer Nedelsky & Arlene W. Saxonhouse & Carola Weil & Saladin Malik Ambar \\
\hline Catharine Newbury & Kenneth F. Scheve & Eric D. Weinstein & Jennifer Amyx \\
\hline Lloyd G. Nigro & Edward Schneier & Madeleine White & Anthony A. Anderson \\
\hline William Niskanen & Morton Schoolman & Leo A. Wiegman & Ingrid D. Anderson \\
\hline Susan S. Northcutt & Joseph M. Schwartz & Eddie N. Williams & Stanley V. Anderson \\
\hline Shayla C. Nunnally & James C. Scott & Angelia R. Wilson & Krister P. Andersson \\
\hline William L. Oakley & H. P. Secher & Elizabeth R. Wingrove & Gloria A. Anthony \\
\hline David M. O’Brien & Mary C. Segers & Kenneth Alan Wink & Connie Anthony \\
\hline Lord Norton of Louth & Ian Shapiro & Patrick J. Wolf & Andy L. Aoki \\
\hline Thomas R. Oliver & Donald Share & Thomas P. Wolf & Paul C. Apostolidis \\
\hline Sima R. Osdoby & Todd C. Shaw & Laura R. Woliver & Murillo de Aragão \\
\hline James L. O’Sullivan & Henry A. Sheinkopf & Gerald C. Wright & Asher Arian \\
\hline General Michael P. Fleming & Kenneth A. Shepsle & J. Mark Wrighton & \\
\hline
\end{tabular}


Hadley Arkes

James Armistead

Paul E. Arnold

Howard Arnould

Leonardo R. Arriola

Kenneth J. Arrow

Graeme Auld

Tom Bacon

Isaac D. Balbus

Thomas J. Baldino

Joseph J. Balitewicz

David Banks

Benjamin R. Barber

Jay Barth

Leah Bassel

Sharon L. Basso

Joyce A. Baugh

Brady P. Baybeck

Staci L. Beavers

Karen Beckwith

Arnold Beichman

Wendell A. Bell

Stephanie L Bellar

David J. Bellshaw

Jane Bendix

Jane Bennett

Frederic A. Bergerson

Michael B. Berkman

David R. Berman

William D. Berry

Daniel R. Biggers

Terri Bimes

R. Kenton Bird

Donald Bishop

Pamela Blackmon

Patricia Boling

Catherine Boone

W. James Booth

Elizabeth T. Boris

Peter J. Bowman

Brent D. Boyea

Karl R. Braithwaite

Jennifer N. Brass

Sheryl Demorest Breen

Christine Thurlow Brenner

Evelyn S. Brewster

William L. Bridgman

Alan Brodman
Allan R. Brown

Benjamin Brown

Victor Bruce

Kent M. Brudney

Anthony R. Brunello

Jonathan R. Bruno

Susan R. Burgess

John Francis Burke

Ross E. Burkhart

Craig M. Burnett

Margaret Burns

Frances G. Burwell

Kenneth G. Busch

Aaron B. Butler

John W. Caldwell

Ernesto F. Calvo

Joseph Cammarano

David E. Campbell

Andrea Louise Campbell

Colin Campbell

Joseph H. Carens

John M. Carey

Elliot W. Carlson

Ellen Carnaghan

R. Charli Carpenter

Austin Carson

Walter Thomas Casey II

N. Joseph Cayer

Kanchan Chandra

Tyson Chaney

Nick Cheesman

Barbara Ann Chotiner

Carmen J. Chung

Jennifer Hayes Clark

Amanda Clayton

Richard A. Clucas

William B. Cody

Cary Coglianese

Howard A. Cohen

Jeffrey E. Cohen

Cathy J. Cohen

Sally S. Cohen

Kenneth M. Coleman

Michael W. Combs

Deirdre M. Condit

Margaret R. Connelly

Pamela Johnston Conover

Charles E. Cook, Jr.
Michael J. Coppedge

Ross J. Corbett

J. Kevin Corder

Cristina Corduneanu-Huci

David Corvette

Joseph W. Cover

Katherine J Cramer

Neta C. Crawford

Portal Pine Creek Canyon

Ingrid Creppell

Michael Crespin

Lyn Loyd Creswell

Joseph Cropsey

William J. Crotty

Jose E. Cruz

John Alexander Curiel

Mark C. Curtis

Lesley Curtis

Christopher P. Dallas-Feeney

Fred R. Dallmayr

Charles U. Daly

Alfonso J. Damico

Rafaela Dancygier

David J. Danelski

James L. Danielson

Charles Robert Davidson

Heath Fogg Davis

R G. Davis

David W. Davis

Rebecca E. Deen

David M. Dehnel

Peter deLeon

Joel H. Delofsky

Kathryn G. Denhardt

John Dinan

Donna Robinson Divine

Richard E. Dodson

Kathleen Dolan

David P. Dolowitz

John Andrew Donaldson

Todd Donovan

David B. Dorman

Jennifer J. Douglas-Abubakar

Douglas C. Dow

Francis J. Duggan

Claudia Hastings Dulmage

Thomas L. Dumm

Pat M. Dunham
Laurel Eckhouse

Peter B. Edelman

James N. Edelman

Catherine R. Edwards

Richard C. Elling

Omar G. Encarnacion

Harold E. Engle

David F. Ericson

Robert S. Erikson

Paulina Ochoa Espejo

Laura E. Evans

C. Lawrence Evans

Diana Evans

Alfred B. Evans, Jr.

Jana Everett

Angelo Falcon

Robert K. Faulkner

Margaret Kent Feczko

Maureen C. Feeley

Paul Ferber

James W. Fesler

Elliott Fiedler

Alexandra Filindra

Peter Graham Fish

James S. Fleming

Richard C. Fording

Richard L. Fox

Peter L. Francia

Lorraine W. Frank

Jason Frank

Robert J. Franzese, Jr.

Jaime Baeza Freer

Siegrun Fox Freyss

Robert S. Friedman

Amy Wallerstein Friedman

Ester Rachel Fuchs

Lee Ann Fujii

Kichizo Fujimoto

Archon Fung

Paul J. Furiga

Henry C. Galant

Gerald Gamm

Judith A. Garber

Raquel Fernandez Garcia

Marcela García-Castañon

Herbert Garfinkel

Thomas R. Genton

Michael Gerard 
Clark C. Gibson

Ernest D. Giglio

Martin Gilens

Micheal W. Giles

Emily R. Gill

Andra Gillespie

Rachel M. Gillum

Terry Gilmour

Holly T. Goerdel

Brian David Goldberg

Sheldon Goldman

Louis W. Goodman

Nicole A. Gordon

Peter A. Gourevitch

Joanne Gowa

Mark A. Graber

Claus Gramckow

Jim Granato

Wyn P. Grant

Amelia Hoover Green

Matthew N. Green

Hannah S. Greenberg

Scott L. Greer

Joseph M. Grieco

Lewis K. Griffith

Colleen M. Grogan

Kimberly A. Gross

Terrie R. Groth

Martin Gruberg

Anna M. Grzymala-Busse

Catherine Blanche Guisan

Dagmar Gunther-Stirn

Michael K. Gusmano

Mary E. Guy

Hans J. Hacker

Steven H. Haeberle

Donald H. Haider

Donald P. Haider-Markel

Kathleen Hale

Drew Philip Halevy

Todd Haltiner

Mary R. Hamilton

Lori Cox Han

Ange-Marie Hancock

Joseph F. Harkins

Shiela Harmon-Martin

Jean Wahl Harris

Lawrence Harrison
Patricia Harte

Roger E. Hartley

Brian Erskine Harvey

Akira Hattori

Lori J. Hausegger

Chip Hauss

Jennifer P. Haydel

Robert L. Healy

Andrew Healy

John P. Heinz

Francis H. Heller

Paul R. Hensel

Mark J. Herbst

Richard Herrera

Ben Highton

Kelly C. Hill

Leslie I. Hill

H. N. Hirsch

Julius W. Hobson, Jr.

Daniel J.B. Hofrenning

Robert E. Hogan

Kay S. Holcombe

Kenneth R. Hoover

Yusaku Horiuchi

Randolph C. Horn

Stephen Horn

R Gordon Hoxie

Margaret S. Hrezo

Sara Hughes

Andy Humm

Constance C.T. Hunt

Bonnie P Hurst

Leann Hurst

Susan D. Hyde

Gary Hymel

Joseph Zikmund, II

Charles Stewart, III

Jack Turner, III

Matthew M. Incera

Helen M. Ingram

Candace Itow

Yoshikazu Iwabuchi

S. Suzan J. Harkness

Alice M. Jackson

Joshua Jansa

Maiah Jaskoski

Amy E. Jasperson

Sherry B. Jeffe
Mary Jeffers

Jennifer M. Jensen

Jack Johannes

Roberta Ann Johnson

Jocelyn M. Johnston

Seth Jolly

Mary Donovan Jones

Roy Parker, Jr.

Hanes Walton, Jr.

Debra Ann Jung

Zehra F. Kabasakal Arat

Ronald Kahn

Kristin Kanthak

Diana Kapiszewski

Christopher F. Karpowitz

Junko Kato

Ellis Katz

Derek Kauneckis

Dimokritos Menelaos

Kavadias

Thomas A. Kazee

Richard C. Kearney

Francis J. Keenan

Lael R. Keiser

Christopher Kelaher

Christopher B. Kenny

Andreas Kern

Samuel Kernell

Cornelius M. Kerwin

Vincent B. Khapoya

Mae C. King

Gary King

Loren King

George M. Kingman

James L. Kingsland

Maureen Kirchhoff

Jennet Kirkpatrick

Jason F. Kirksey

John J. Kirlin

Kathleen Knight

Hidetaka Kobayashi

Matthew A. Kocher

Isaac A. Kramnick

Henry Krisch

Jonathan Kropko

Melvin T. L. Ang

Richard Labunski

Marc Landy
Joseph H. Lane, Jr.

Thomas S. Langston

Joseph LaPalombara

Edward L. Lascher, Jr.

Thelma Lavine

J. Celeste Lay

Lan Thuc Le

Matthew Lebo

Susan S. Lederman

Chinyelu Kambui Lee

Taeku Lee

Thomas J. Leeper

Carol Skalnik Leff

Jerome S. Legge, Jr.

Valerie Lehr

Janet E. Leigh

Lance T. LeLoup

Arthur J. Lerman

Michael H. Levin

Nanette S. Levinson

Meira Levinson

Naomi Levy

Peter M. Lewis

Samer abu Libdeh

Roy Licklider

Jethro K. Lieberman

Carl Lieberman

Sarah F. Liebschutz

Michael Lienesch

Alfred R. Light

Thomas W. Likens

Charles E. Lindblom

Keena Lipsitz

Soledad Loaeza

Marvin R. Loewy

Dianne N. Long

Andrea M. Lopez

Rodrigo Losada

Diane Love

Nicholas P. Lovrich, Jr.

Joseph E. Lowndes

Julia Lynch

David A. M. Peterson

Roderick MacFarquhar

Michael B. MacKuen

Stuart A. MacNiven

Amanda M. Maher

James Mahoney 


\begin{tabular}{|c|c|c|c|}
\hline Kathleen A. Mahoney-Norris & Eric Morgenstern & Richard L. Pacelle, Jr. & Rob Reich \\
\hline Samantha Ann Majic & Valerie Ona Morkevicius & Harry P. Pachon & Beth Reingold \\
\hline Todd Makse & Lorenzo Morris & Elizabeth Paddock & Howard L. Reiter \\
\hline Michael J. Malbin & K. C. Morrison & Marian Lief Palley & Russell D. Renka \\
\hline Edmund J. Malesky & Charles C. Moskos, Jr. & Zhengqi Pan & Stanley A. Renshon \\
\hline Harvey C. Mansfield & Ken Moskowitz & Christopher S. Parker & Joao Resende-Santos \\
\hline Paul Christopher Manuel & Jim Moss \& Company & Bruce Parrott & Norma M. Riccucci \\
\hline Andrew F. March & Burton B. Moyer, Jr. & Janine A. Parry & Ross R. Rice \\
\hline Patchen Markell & Donald P. Moynihan & Samuel C. Patterson & Lilliard E. Richardson, Jr. \\
\hline Andrei S. Markovits & Susanne D. Mueller & Thomas E. Patterson & Julia T. Richie \\
\hline Andrew D. Martin & Russell Muirhead & Arthur C. Paulson & Irelene P. Ricks \\
\hline Sharon Mastracci & William D. Muller & Jeffrey S. Peake & Elizabeth Rigby \\
\hline Donald J. Matthewson & Costanza Musu & Shanna Pearson-Merkowitz & Alice Robbin \\
\hline Russell Mayer & Tun Myint & Edward Lewis Pederson & Suzanne M. Robbins \\
\hline Rick Mayes & Emily Nacol & Ann Pejsa & James C. Roberts \\
\hline Steven Maynard-Moody & Akiko Nakamura & Pamela D. Pelletreau & Margaret A. Robinson \\
\hline Mark G. Mazzie & John Nalbandian & Bruce Pencek & Scott E. Robinson \\
\hline Wendy McCorkle & Clayton M. Nall & Thomas Pepinsky & Maya Rockeymoore \\
\hline John P. McCormick & Doron Navot & J. Salvador Peralta & Robert R. Rodgers \\
\hline Ora McCreary & Vernon R. Neeece & S. Peregrino & John E. Roemer \\
\hline H. Coleman McGinnis & Laura Neish & Clarisa Perez-Armendariz & Susan Carol Rogers \\
\hline Michael D. McGinnis & Michael J. Nelson & Anthony D. Perry & Jon C. Rogowski \\
\hline Benjamin McKean & Aram Nerguizian & Ravi Kumar Perry & Meredith Rolfe \\
\hline Joseph Paul McLaughlin, Jr. & Kathryn Newcomer & Jack Pfister & William J. Ronan \\
\hline Joan E. McLean & Charldean Newell & Brian J. Phillips & James N. Rosenau \\
\hline Scott L. McLean & David Newman & John Charles Pierce & Marie B. Rosenberg \\
\hline Leslie B. McLemore & Richard Nielsen & Neil Andrew Pinney & Raymond A. Rosenfeld \\
\hline Lois I. McMullen & Daniel L. Nielson & Richard M. Pious & Margaret Ann Rosenthal \\
\hline Jillian Medeiros & Lela G. Noble & Michael Callaghan Pisapia & Cindy Simon Rosenthal \\
\hline David Menefee-Libey & Joseph L. Nogee & Leonid Platonov & Howard Rosenthal \\
\hline Arthur E. Menna & Barbara Norrander & Jerry L. Polinard & Marc Howard Ross \\
\hline Tamara Metz & Noelle Helen Norton & Linda O. Polsby & Lee Ross \\
\hline Wanda F. Meyer-Price & Anne Norton & Marsha Pripstein Posusney & Brandon Rottinghaus \\
\hline Kevin J. Middlebrook & Grady H. Nunn & Stephen Poulson & Francis E. Rourke \\
\hline Sidney M. Milkis & Malliga Och & Adam Przeworski & Blair A. Ruble \\
\hline Mark C. Miller & Elizabeth A. Oldmixon & Steven R Putansu & Ricardo Antonio Ruiz de la \\
\hline Susan Marie Miller & Mark Oleszek & Tracy L. R. Lightcap & \\
\hline Harris N. Miller & Susan M. Olson & Eric W. Rader & Robert C. Sahr \\
\hline Gabriel Miller & Laura R. Olson & Wendy M. Rahn & Motohide Saji \\
\hline Helen V. Milner & Molly O’Neal & Hal G. Rainey & William G. Sale \\
\hline Jeffrey Milyo & John M. Orbell & Ronald B. Rapoport & Anthony A. Samad \\
\hline James Andrew Mitchell & Mitchell A. Orenstein & Sharon LaWanda Rapport & Jose Ramon Sanchez \\
\hline Carla E. Molette-Ogden & D’ Andra Orey & Jorgen S. Rasmussen & Andrea Stevenson Sanjian \\
\hline John Hull Mollenkopf & Frederick K. Orkin & John David Rausch, Jr. & Eric Schickler \\
\hline Lisa J. Montoya & Tracy Osborn & James H. Read & Steven E. Schier \\
\hline Celeste M. Montoya & Chieko Kitagawa Otsuru & David P. Rebovich & Andrew J. Schlewitz \\
\hline Richard M. Moose & Scott Otteman & Mavis Mann Reeves & Patricia Schlueter \\
\hline David R. Morgan & Michael Leo Owens & Andrew Rehfeld & John R. Schmidhauser \\
\hline
\end{tabular}




\begin{tabular}{|c|c|c|c|}
\hline Vivien A. Schmidt & Robert L. Spurrier, Jr. & Sean W. Twombly & Steven I. Wilkinson \\
\hline Mark L. Schneider & Peverill Squire & Frank J. Tysen & Stephanie Lynn Williams \\
\hline Anne L. Schneider & Joseph L. Stager & Isaac Unah & Kim M. Williams \\
\hline Cheryl M. Schonhardt-Bailey & Manfred B. Steger & Otto F. Unsinn & Jonathan Winburn \\
\hline Scot Schraufnagel & Michael Bernard Stein & Ali A. Valenzuela & Christopher M. Witko \\
\hline Nancy L. Schwartz & Carl W. Stenberg III & Renee B. Van Vechten & Deborah A. Wolf \\
\hline Melissa A. Schwartzberg & Otis H. Stephens & R. Lawrence Vandenberg & Matthew D. Wolf \\
\hline Carl E. Schwarz & Susan M. Sterett & Kenneth L. Verdoia & Sheldon S. Wolin \\
\hline Robert Scigliano & Arthur R. Stevens & Yaacov Y.I. Vertzberger & Stephen B. Wood \\
\hline John E. Seery & George Stickles & Jill Mccalla Vickers & Michael J. Woodruff \\
\hline Harold Seidman & Walter J. Stone & Robert Vitalis & Neal Woods \\
\hline Paul J. Seligman & Jeffrey D. Straussman & Helen R. Voss & Richard Worthington \\
\hline Robert Serec & Mervin K. Strickler, Jr. & Jeremy Waldron & Mark Wynn \\
\hline Jeffrey G. Seward & Philippa Strum & David B. Walker & Tao Xie \\
\hline Byron E. Shafer & Henry Suhr & Lee D. Walker & Takakazu Yamagishi \\
\hline D. Michael Shafer & Terry Sullivan & Thomas G. Walker & John Yang \\
\hline David M. Shafie & Todd Swanstrom & Sophia Jordan Wallace & Dvora Yanow \\
\hline Kenneth Sharpe & Rex J. Swartz & Herbert Waltzer & Carolina Ferrerosa Young \\
\hline Tommie Shelby & Martin J. Sweet & Julian Wamble & Barry Zalcman \\
\hline Mack C. Shelley II & Ann Swidler & Yanlai Wang & Anne B. Zill \\
\hline Doh C. Shin & Ivan Swift & Adam L. Warber & James M. Zoetewey \\
\hline Carolyn M. Shrewsbury & John J. Szmer & Michael D. Ward & Aristide R. Zolberg \\
\hline W. Scott Shrewsbury & Mina Tadrus & Robert E. Ward & Alan S. Zuckerman \\
\hline Steven A. Shull & Lorraine M. Tafoya & Christopher Warshaw & Catherine H. Zuckert \\
\hline Jeffrey Sikkenga & Joseph M. Tages & Omar Wasow & The Freedom Forum \\
\hline Elaine E. Silverman & Jordan Tama & Price I. Watkins & Girls Ranch, Inc. of Arizona \\
\hline Sheldon W. Simon & A. Tannenbaum & Wendy Lyn Watson & Lynne Rienner Publishers \\
\hline Lewis B. Sims & Raymond Tanter & Maria-Rosa Watson & Pfizer Inc. \\
\hline J.P. Singh & Kathleen Tapport & William L. Waugh, Jr. & Princeton University \\
\hline Henry B. Sirgo & Nathan Tarcov & Vesla Mae Weaver & Sage Publications \\
\hline Stephen Skowronek & Melinda Rae Tarsi & David J. Webber & \\
\hline Royal Daniel Sloan, Jr. & Mark Zachary Taylor & Marc D. Weiner & CONTRIBUTORS \\
\hline Corwin E. Smidt & A. Stephen Telkins & Meredith L. Weiss & Sobello \\
\hline Daniel A. Smith & Leslie Paul Thiele & David M. Welborn & Sabella Ogbobode Abidde \\
\hline Kevin B. Smith & Darryl Clark Thomas & Andreas Wesserle & CIIIstopher П. АС̆еI \\
\hline Alan G. Smith & Emma S. Thomas & Ellis M. West & Miartna A. Ackeisoerg \\
\hline Elizabeth S. Smith & Craig W. Thomas & Bruce Western & George г. Ackemman \\
\hline Stephen Samuel Smith & Laurence Thomas & Marcia Lynn Whicker & Marsha E. Ackermann \\
\hline Candis Watts Smith & Sue Thomas & Gordon P. Whitaker & Derijartm Mcosta \\
\hline Robin Smith & Joan Hulse Thompson & Ismail K. White & Inomas w. Adams \\
\hline Robert Smith & Frank J. Thompson & Stephen L. White & \\
\hline Leonid V. Smorgunov & Shatema Threadcraft & Terri White & Claire Leslie Adida \\
\hline Albert Somit & Kurt M. Thurmaier & Sharon G. Whitney & Kobert Agranon \\
\hline Sarah Song & Betty Tillman & Bonnie Smith Whyte & Akmal Aleem \\
\hline June S. Speakman & Leslie A. Tourigny & Erik M. Wibbels & Julip llayeres \\
\hline Roy A. Speckhard & Brian C. Turner & Lt. Col. Donald Wickler & Jul1o Alqueres \\
\hline Leonard M. Sperry, Jr. & Robin L. Turner & Martha Wilfahrt & Bruce F Altschuler \\
\hline Jeff Spinner-Halev & Dale Turner & Curtis C. Wilkie, Jr. & 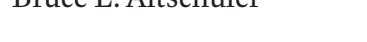 \\
\hline
\end{tabular}


Joseph Alulis

Sulaiman Alwahid

Ellen Ann Andersen

Greg Anderson

Kevin Anderson

Stefan Andreasson

Yuen Yuen Ang

Gloria C. Anglon

Jacquelyn A. Anthony

Ciro Antignani, Jr.

Jeff Paul Antsen

Dominique Apollon

Harriet B. Applewhite

Janni L. Aragon

Kiichiro Arai

Rogério Bastos Arantes

Kathleen M. Araujo

Phillip J. Ardoin

Luisa Maria Arevalo

Shelly R. Arsneault

Raka Arya

Koki Theodore Asakura

Victor Asal

Michael A. Ashburn

Athanasios Augoustatos

Sharon Denise Austin

Olga A. Avdeyeva

George Avelino

Claudia N. Avellaneda

Richard Avramenko

Regina Axelrod

Brent E. Ayer

Bonnie Ayodele

Alice D. Ba

Carl Baar

Jason Badura

Catie Snow Bailard

Jennifer L. Bailey

Kimberly Bakeberg

John R. Baker

Nancy V. Baker

Terence Ball

Robert A Ballingall

Navin A. Bapat

Scott Barclay

Derek Barker

Twiley W. Barker, Jr.

Ankita Barman
David M. Barrett

Elizabeth Barringer

Sharon A. Barrios

Michael J. Barron

Robert Barsley

Trina Kay Barton

Heather Bastedo

Richard M. Battistoni

Robert F. Bauer

Bruce Baum

Donald C. Baumer

Nidia Bautista

Glenn Beamer

Ronald J Beaton

Emily Beaulieu

Matthew N. Beckmann

Nancy L. Bednar

Franz Beitzinger

Patrick M. Bell

Laura N. Bell

Laid Bendadoud

Robert Earl Bender

Liza Abram Benham

Kenneth R. Benoit

Luke Angelo Bergamini

Benjamin F. Berger

Allison Berland

Eli Berman

William T. Bernhard

Nancy Berson

Wallace H. Best

Erik Charles Beuck

Benjamin Bierly

Michael Binder

Stephen Bird

Nathaniel A Birkhead

Thomas A. Birkland

Robyn M. Bishop

Samuel Bishop

Bidisha Biswas

Kelvin Calhoun Black

Lavonna J. Blair Lewis

Charles H. Blake

Randall W. Bland

Sarah Bland

John W. Blaney

Rebecca E. Blanton

Eric R. Boehme
Charles R. Boehmer

Alison E. Bolt

Thomas J. Bond

Frank Bonilla

Thomas E. Borcherding

Fabian A. Borges

Craig Borowiak

Jocelyn M. Boryczka

Linda Bosniak

Aimée Bourassa

Philip Duncan Bousquet

Helen G. Boutrous

Patricia R. Bowley

Daniel Bruce Braaten

Karl R. Braithwaite

Dawn Brancati

Gildo Marcal Brandao

Pamela T. Brannon

Kathleen A. Bratton

Amanda Jean Brazier

John Brehm

Marla Brettschneider

Marijke Breuning

Alan David Breward

Dave Bridge

Reuben E. Brigety II

Kel Jacobs Britvec

Nailah Brock

Evelyn Z. Brodkin

Nick Bromell

Roger A. Brooks

Deborah Jordan Brooks

Nadia E. Brown

Ronald E. Brown

John E. Brown

Kevin James Brown

Colin M Brown

Lara Michelle Brown

Adam R. Brown

John R. Brown

Kelley S. Brown

Rufus P. Browning

J. Lawrence Broz

Adeyinka Ayobambo Bruce Omotunde

Claudia Franziska Bruehwiler Haeusermann

Jodi Bruhn
Daniel Brumberg

Lisa A. Bryant

J. Vincent Buck

John E Budzinski

Eloise A. Buker

Mlada Bukovansky

Fran Lisa Buntman

Cynthia Burack

Barry C. Burden

Thawilwadee Bureekul

Thomas F. Burke

Walter Dean Burnham

Timothy W. Burns

Peter Burns

Carolee Bush

Elizabeth Bussiere

Charles E. Butterworth

Eric Buzzetti

Keith J. Bybee

Deanna Caldwell

Direlle R. Calica

Pamela Camerra-Rowe

Carlo Caparruva

Teri L. Caraway

Christopher M. Cardona

Jessica D. Carew

Douglas Carlisle

Elizabeth C. Carlson

Sara G. Carpenter

Nancy Carrillo

R. Wesley Carrington

Berenice A. Carroll

Niambi M. Carter

Brenda W. Carter

Stephanie Carvin

Leo E. Casey

K. Lynn Cates

Thomas P. Cavanna

Matthew M. Caverly

James W. Ceaser

Mwita Chacha

Stefanie Chambers

Maurice B. Champagne

Steve Charnovitz

Naomi Chazan

Rumi Chen

Francis Cheneval

Michelle L. Chin 
Jiyoung Cho

Robert C. Chope, Jr.

Tom Christensen

Jeffrey Church

Allan J. Cigler

Kelly Clancy

Caleb M. Clark

Ann Marie Clark

Terry Nichols Clark

Joan B. Claybrook

Frank R. Cleminson

Ajenai S. Clemmons

David Clinton

Jean Clipperton

John Cluverius

Joseph Cobetto

J. Patrick Coby

Frank S. Cohen

Joshua Cohen

Gloria Cohen-Dion

Daniel Cohn

Guy C. Colarulli

Leonard A. Cole

Sylvester (SC) Coleman

John Coleman

Austin College

Kenneth Collier

Loren Collingwood

Freddie C. Colston

Anand Bertrand Commissiong

Mary Ann Compton

Carol B. Conaway

Tim Conlan

Edward Steven Connell

Luis M Contreras

Pamela Y. Cook

Catherine M. Cooney-Mesker

Rosalyn Cooperman

Gary W. Copeland

Robert E. Corkran

Michelle Cornelius

Javier Corrales

Jeronimo Cortina

Thomaz G. Costa

Charles L. Cotrell.

Cornelius P. Cotter

John W. Cotton

Margery M. Coulson-Clark
Matthew W. Courser

Skylar Covich

George H. Cox

Thomas Craemer

Sue E.S. Crawford

Nyron N. Crawford

Kevin Croke

Jesse M. Crosson

Jason A. Crotty

Don W. Crowley

Jose Miguel Cruz

Paul J. Culhane

Michael Stuart Cummings

Timothy W. Cunningham

Richard T. Cupitt

Paisley Currah

George J. Cvejanovich

Charles A Dainoff

Tom Darby

Paolo Dardanelli

Carol Dauda

Eugene Davidson

Donn G. Davis

Matthew K. Davis

Linda Davis

Renee Noelle Day

Isabel Ruiz de Castañeda

Thomas S. De Luca, Jr.

Leany Barreiro de Sousa Lemos

Matthew DeBell

Melissa Deckman

Jane S. DeHart

Eric van der Vort

Christopher D. DeSante

Bruce Desmarais

David Dessler

Dieter Dettke

Bryan J. Dettrey

Mark G. Detweiler

Louis Diaco

John Michael Diamond

Larry Diamond

Jennifer Segal Diascro

Christopher W. Diaz

Alberto Diaz-Cayeros

Charles K. Dilley

Michelle L. Dion
Kristin Smith Diwan

Daniel J. Doherty

Jameson W. Doig

Rhonda Dolen

Stefan P. Dolgert

Nelson C. Dometrius

Jose Miguel Dominguez

Jaime Dominguez

Thomas J. Donahue

Richard F. Doner

Matthew Doppke

Sandra Lee Dorociak

Pedro G. dos Santos

Paul Dosh

Charles W. Dougherty

James W. Douglas

Rebekah Dowd

William M Downer

Victoria Ann Doyon

Alex N. Dragnich

Anne L. Draznin

Jennifer Raymond Dresden

John S. Dryzek

Yusi Du

Jane Duckett

Sean P. Duffy

Thad Dunning

Denise E. Dutton

Angela Doll Dworin

Diana Dwyre

Bradley F. Dyke

Gregory Eady

Valerie Earle

David C. Earnest

Bianca Easterly

Cheryl L. Eavey

Alan O. Ebenstein

John Echeverri-Gent

Carlene Edie

Ruth M. Ediger

Sean D. Ehrlich

Marianne Eichelberger

Johannes Eijmberts

Robert M. Eisinger

Joyce A.M. Ejukonemu

Sarah L Elichko

Ginger Leigh Elliott-Teague

Cali Mortenson Ellis
R. Amy Elman

Deborah K. Elms

David Endicott

Stephen M. Engel

Michael J. Ensley

Charles R. Epp

Arlen I. Erdahl

John G. Eriksen

Maria C. Escobar-Lemmon

Adetoun Oluwaremilekun

Esther Adenuga

Lloyd S. Etheredge

J. Peter Euben

Kamilu Sani Fage

Hesham Faied

Pamela S. Falk

Jason Falvey

Rick D. Farmer

David M. Farrell

Henry Farrell

Stephen M. Feldman

Botond Feledy

Kathy E. Ferguson

Margaret R. Ferguson

Kenneth E. Fernandez

Sergio Fernandez

Pablo Fernandez-Vazquez

Isabel Anunciacao Ferraz Camisao

Sandra Leonie Field

Bonnie N. Field

Edwin H. Fields

Lynn Fields

John W. Finch

Terence T. Finn

Joseph Fischel

Alexander Christoph Fischer

Steve Fish

Samuel H. Fisher III

Robert M. Fishman

J. Samuel Fitch

Roy B. Flemming

John A Fliter

Jackson B Floyd

Judy Casburn Floyd

Edmund Fong

Ware Fong

Robert Ford 


\begin{tabular}{|c|c|c|c|}
\hline Michael David Forrest & Rodney Wade Gill & Samantha Guthrie & Yoshiko M. Herrera \\
\hline Margaret J. Foster & Jonathan Githens-Mazer & Dean Eugenio Guzman & Frederick M. Herrmann \\
\hline Carmen Le Foulon & James M. Glass & Claire Haeg & Dean R. Hewitt \\
\hline Lisa Pullen Foust & Rebecca A. Glazier & Timothy Haglund & Timothy William Hibbard \\
\hline Bernard L. Fraga & Peggy Glick & Michael W. Hail & David B. Hill \\
\hline Pauldine France & Marcia L. Godwin & Susan Haire & Larry B. Hill \\
\hline Sekou Franklin & Col. Robert L. Goerder, Ret. & Kegan Alexander Hakim & Paul T. Hill \\
\hline William W. Franko & Catherine Goetze & Smith & Kelsey Hinchliffe \\
\hline Frank Franz & Larry Gold & Brien Hallett & Samuel M. Hines, Jr. \\
\hline Simon Tobias Franzmann & Leslie Friedman Goldstein & Leon Halpert & Hiroshi Hirano \\
\hline Deen Freelon & Saeid Golkar & Mark Halverson-Wente & Nancy J. Hirschmann \\
\hline Frederick W. Frey & Mark Golub & Roger Hamburg & Carmen Jacqueline Ho \\
\hline Verena Fritz & Vanna Antonia Gonzales & Raymond A. Hamilton II & Bai Linh Hoang \\
\hline Fred M. Frohock & Jorge M. Gonzales Galoffin & Darrell A. Hamlin & Emily Howden Hoechst \\
\hline Timothy Frye & Brenda Nicia Gonzalez Gomez & Dan J. Hansen & Samuel B. Hoff \\
\hline Jonathan J. Fuentes & Naomi W. Goodell & Eric Hansen & Stanley Hoffmann \\
\hline Francis Fukuyama & Jeanette Goodstein & Jeffrey J. Harden & Marie Hojnacki \\
\hline Timothy Fuller & Dariusz Gora-Szopinski & Robert L. Hardmond & Thomas M. Holbrook \\
\hline Elena Gadjanova & Ann Gordon & Christine B. Harrington & Kenneth M. Holland \\
\hline Nicci Gafinowitz & Lilly J. Goren & deBorah Ann Harris & Richard Samuel Holliday \\
\hline Francesca Gains & Wendi Gosliner & Dennis Harris & Thomas T. Holyoke \\
\hline Frances Galante & Kristin Goss & Melissa Harris-Perry & Claudio A Holzner \\
\hline Susan Gallagher & Els de Graauw & Jonathan Hartlyn & Juliet Hooker \\
\hline David H. Galler & Joan Grace & Nancy Hartsock & P. Terrence Hopmann \\
\hline Archibald Galloway & Theresa L Graham & Brian M. Harward & Nadia Rabesahala Horning \\
\hline Paula Daniela Ganga & Lawrence Sherman Graham & Miao-ling Lin Hasenkamp & Germaine A. Hoston \\
\hline Olivia Garcia & Wayne G. Granquist & Vivien Hastings & Peter B. Hovde \\
\hline Rulon R. Garfield & Keneshia N. Grant & Tomohisa Hattori & Lise Morje Howard \\
\hline Alex Garlick & Blake P. Graves & Leah Haus & Tiffiany O. Howard \\
\hline R. Sam Garrett & Maggie Gray & Melissa Haussman & Christopher Howard \\
\hline Jose E. Garriga-Pico & Patrick H Green & Daniel P. Hawes & Susan E. Howell \\
\hline Bryan Garsten & Donald P. Green & Jonathan P. Hawley & Dustin Howes \\
\hline Robin Gaul & Anne Green & Barbara J. Hayler & Vicki Hsueh \\
\hline Raymond Gavins & Jill S. Greenlee & Eugene G Hayunga & Yasheng Huang \\
\hline Judith Gay & Claire Greenstein & Chad Hazlett & Betty G. Hubbell \\
\hline Scott Gehlbach & Ryan D. Griffiths & Jianzi He & Martine Huberty \\
\hline Shamira M. Gelbman & Amanda Grigg & Alice L. Hearst & Larry L. Hudson \\
\hline Myron Genel & Bernard N. Grofman & Kenneth A. Heath & Alexandra Huneeus \\
\hline Tracey E. George & Paul Gronke & Karen Hein & Valerie F. Hunt \\
\hline Steven A Gerencser & Wes Grooms & Caroline Heldman & Jennifer S Hunt \\
\hline Daniel B. German & Justin H. Gross & Caroyn Hendriks & Robert E. Hunter \\
\hline Heather Getha-Taylor & Seth A. Grossman & Laura A. Henry & Kate Carney Huston \\
\hline Arash Ghafouri & Guy Grossman & Beth M. Henschen & Michael A. Iasilli \\
\hline Michael T. Gibbons & Gerard S. Gryski & Michelle E Hentz & Rasidi Abiodun Ifafore \\
\hline Dan Gibson & Nelson P. Guild & Ricardo Sucre Heredia & Rodolfo Espino, III \\
\hline Anthony "Jack" Gierzynski & Alexandra Guisinger & Charles F. Hermann & Philip Ingrassia \\
\hline Michael Jaye Gilbreath & Jeff Gulati & $\begin{array}{l}\text { Jose Antonio Hernandez } \\
\text { Company }\end{array}$ & Giovanni Ingrosso \\
\hline Murray Gilford & Charles David Gustafson & & Mackenzie Leigh Israel- \\
\hline
\end{tabular}




\begin{tabular}{|c|c|c|c|}
\hline Trummel & Sabrina Karim & Douglas L. Koopman & Yuen-Ching B. Lee \\
\hline Ivan Dinev Ivanov & Jason S. Kassel & Kyle Casimir Kopko & Caitlin Harrington Lee \\
\hline Misako Iwamoto & Mark J. Kaswan & Fae L. Korsmo & Aleksandra Sznajder Lee \\
\hline Antoine M. Jackson & Lyn Kathlene & Liane C. Kosaki & Ji Yun Lee \\
\hline Alan M. Jacobs & Koji Kato & Laura Kosch-Cocozzella & Gail Leftwich \\
\hline Ambrous Jacobs & Ruth D. Kaufman & Hanako Koyama & Jeffrey W. Legro \\
\hline Matthew L. Jacobsmeier & Joyce P. Kaufman & John F. Kozeletz & Fabrice Lehoucq \\
\hline Joshua Alec Jaeschke & Herbert Kaufman & Vitaly Kozyrev & Debra Lynn Leiter \\
\hline Erwin A. Jaffe & Kerim Can Kavakli & Sina Jo Kramer & Jean D. Lemasurier \\
\hline Jane S. Jaquette & Karen M. Kedrowski & Robert P. Kraynak & Jonathan Lemco \\
\hline Chris Jarmon & William J. Kelleher & Rebecca J. Kreitzer & Marc Lendler \\
\hline Khoshaba Jasim & Ann C. Keller & Ladis K.D. Kristof & Harry A. Lenhart \\
\hline Christina F. Jeffrey & Jonathan Keller & Paul F. Kromer & Meng U Leong \\
\hline Shannon Jenkins & Judith Kelley & Seldon M. Kruger & Stephan Lesher \\
\hline Bruce Jennings & J. Edward Kellough & Dmytro Roman Kulchitsky & David Lessard \\
\hline Benjamin M. Jensen & Christine A. Kelly. & Sloane Rachael Kuney & Elaine S. Levine \\
\hline Andres E Jimenez & Steven Kelts & Todd Kunioka & Traci M. Levy \\
\hline Michelle E. Johns & Edward J. Kelty & Susan M. Kunkle & Michael Alan Lewis \\
\hline Ollie A. Johnson & Frederick Kent & Karl T. Kurtz & Jeffrey B. Lewis \\
\hline Lori A. Johnson & Lane Kenworthy & Edward Kwon & Paul G. Lewis \\
\hline Douglas B. Johnson & Nannerl O. Keohane & Izul de la Vega & Jeremy R.T. Lewis \\
\hline Martha C. Johnson & John E. Kern & Dean P. Lacy & Jacob S. Lewis \\
\hline Vernon D. Johnson & Kouslaa Kessler-Mata & Marianne Lado & Michael S. Lewis-Beck \\
\hline Mark L. Johnson & Gilbert M. Khadiagala & Gordon Lafer & Steven B. Lichtman \\
\hline Paul M. Johnson & Dwight C. Kiel & Crystal LaGrone & Robert C. Lieberman \\
\hline Kimberley S. Johnson & Christina Kiel & Chryl Laird & Phoebe S. Liebig \\
\hline Avra Johnson & Kent J. Kille & Andrew Lamas & Staffan I. Lindberg \\
\hline Mark Johnson & Jung Ook Kim & Alan C. Lamborn & Earl W. Lindveit \\
\hline W. Lee Johnston & So Young Kim & Thomas D. Lancaster & Juan J. Linz \\
\hline Patrick S. Johnston & Doo-Rae Kim & Pierre F. Landry & Michael Lipscomb \\
\hline Jessica Alexis Jolicoeur Rich & David C. Kimball & Robert W. Lane & Charles Lipson \\
\hline Jessica L Jones & Robert J. King & Carol Langdon & Harris M. Lirtzman \\
\hline Bradford S. Jones & Aaron R. King & Stephen Lange & Rebecca Lisi \\
\hline Cara Jones & Vanessa King & Vickie Langohr & George T. Little \\
\hline Murel M. Jones, Jr. & Rebecca Kingston & Christopher W. Larimer & David William Livingstone \\
\hline Jason Jordan & Niki Kirkby & Leah Larson-Rabin & Flordeliz Llarena \\
\hline Padi Hallam Joseph & Patricia Kirkland & Narayani Lasala-Blanco & Jill L. Locke \\
\hline Maya Joseph & Sue W. Kirkpatrick & Daniel Laurison & Erin Lockwood \\
\hline Charlotte Joseph & Knut Kirste & William Tricot Laventhal & Dennis E. Logue, Jr. \\
\hline Jyl J Josephson & Elizabeth E. Kiss & Regina G. Lawrence & Jinee Lokaneeta \\
\hline Peter Josephson & James Kewir Kiven & Jeffrey R. Lax & S. Hansen Long \\
\hline Carlos E. Juarez & Casey A. Klofstad & Laureen Lazarovici & David Lopez \\
\hline Diana M. Judd & Robert D. Knight & Loan K. Le & Diqing Lou \\
\hline Dennis R. Judd & Stephen A. Kocs & Victor T. Le Vine & Daniel H. Lowenstein \\
\hline Zinaida Kally & Vladimir Kogan & Adrienne LeBas & Patrick Lown \\
\hline Rumen Kanchev & Thomas S. Kolasa & Christopher Lebron & Carol R. Lubin \\
\hline Nicholas Matthew Kandrac & Edward Albert Kolodziej & Liz Lebron & Ellen M. Lust \\
\hline Hye Yun Kang & Tomas Koontz & Fragano S.J. Ledgister & Ian S. Lustick \\
\hline
\end{tabular}




\section{Keith Luter}

Kit G. Machado

James MacHaffie

Michael Kenneth MacKenzie

Paul P. Maeser

Paasha Mahdavi

Julianne Mahler

Daniel J. Mahoney

Barry Mahoney

Elaine P. Maimon

Elena Maltseva

Peter Phillips Mandaville

Ardith L. Maney

Lynne L. Manganaro

Cynthia Massie Mara

Robert H Marchini

Greg Marfleet

Michael Margolis

Nikolay V. Marinov

Walter G. Markham

Elizabeth Markovits

Irving Leonard Markovitz

Melissa J. Marschall

Sherry L. Martin

Jose Roberto Martinez

Helena Martins

Claude Marx

Curtis M. Masiello

John G. Mason

Roger D. Masters

Marsha A. Matson

Elizabeth C. Matto

Vincent Mauro

Sarah Maxey

Jeremy Mayer

Jamie Mayerfeld

Shom Mazumder

Andrea McAtee

Dylan McAuliffe

Margaret M. McBeth

Andrew James McCall

Michael W. McCann

Allan L. McCutcheon

Eric L. McDaniel

Alexis McGill

Adam McGlynn

Mary A. McHugh

David McIlvain
Amy Melissa McKay

Seth C. McKee

Lynn McKee

Clyde D. Mckee, Jr.

Judith McKenzie

Heather Elko McKibben

Judithanne Scourfield McLauchlan

John McMahon

Roberta Medford

Stephen E. Medvec

Stephen K. Medvic

Scott R. Meinke

James P. Melcher

Mark Mellman

Richard H. Melton

Arthur M. Melzer

Charles E. Menifield

Bryan K. Mercier

Jennifer L. Merolla

Brandon Merrell

Stephen A. Merrill

Martha L. Merritt

Lee Kendall Metcalf

William Mezzetti

Kristin Grace Michelitch

Veronica Michel-Luviano

Joel A. Middleton

Sophia Mihic

Elizabeth B. Miller

Joshua I. Miller

Terry L. Miller

Nicholas R. Miller

Sven Miller

Deborah J. Milly

Michael Minkenberg

Daniel J. Minnich

Michael D. Minta

Karen L. Mitchell

Zlatin Mitkov

Olivier R. Mitterand

Farida Mohammed

Kimberly Moloney

Benoit François Monange

Fred M. Monardi

Mahmood Monshipouri

Tonia R. Moore

Frank O. Mora
Kevin Morales

Andrea L. Morato-Lara

Sarah M. Morehouse

Alejandro Moreno

Margot Morgan

Brendan M Morris

Jennifer Morstein

Eric Seth Mosinger

Karen Mossberger

Julie Mostov

Naomi Moswete

Michelle Moyd

Lisa Mueller

Dipali Mukhopadhyay

Patrick A. Mulloy

Diana Munoz

Naomi Murakawa

Jeffrey Stevenson Murer

Ragnhild Louise Muriaas

Dale D. Murphy

Matthew D. Murray

Robert E. Mutch

Adam Myers

Carol Nackenoff

Brigitte Nacos

Stuart S. Nagel

Tommaso Nannicini

Emma Natal

Sharon Ann Navarro

Dani Nedal

Dalmas H. Nelson

Joan M. Nelson

Jami Nelson-Nunez

Sheila M. Neroda

Gerald Neumark

Joan Hillebrand Neumiller

Lina Newton

Bijoux Gidibu Ngwanda

Cynthia T. Nickel

Katherine Niemiec

Demetra Smith Nightingale

Ron Nikora

Peter Nitschke

Kiyoko K. Nitz

Jill Norgren

Stephen Noriega

Sally Nuamah

Julio Figueroa Nunez
Gilbert David Nuñez

Stephen A. Nuno

Ikenna Jesse Nwoke

Anthony K. Nyame

Sulayman Nyang

Holly Oberle

Anthony Martin O'Halloran

Christopher Ojeda

Stephen Wayne Okel

Naoko Oki

Chika Okoye

Christopher Olds

Brendan O'Leary

Santiago Olivella

Kelly O'Malley

Ido Oren

Candice D. Ortbals

Anna Maria Ortiz

Elizabeth (Betty)

O'Shaughnessy

Mara Cecilia Ostfeld

Mara Ostfeld

Taiwo Owoeye

Olavo L. P. de Carvalho

James S. Pacy

Lwanyo Padiet

Lilian M. Pagan

Anthony R. Pahnke

Daniel Palazzolo

Anthony Palmer

Michael Palmer

Betsy Levy Paluck

Chengxin Pan

Costas Panagopoulos

Alice Pannier

Joshua S. Parens

Joseph B. Parker

Shannon Parker

Daniel J. Parks

James M. Patterson

Ifechukwu Ajuta Paul

Joan Paylo

Darryl C. Payne

Kathryn Pearson

Carl Pedersen

Mark Peffley

John P. Pelissero

Jose Raul Perales 


\begin{tabular}{|c|c|c|c|}
\hline Christian Alexis Perez & Kerry Ratigan & Bernard H. Ross & Beth S. Schapiro \\
\hline Moises Perez, Jr. & James P. Rausch & Robert L. Ross & Denise L. Scheberle \\
\hline Ellis Perlman & Earl C. Ravenal & Jason P. Rouby & Emily Schilling \\
\hline Ayodeji Kamau Perrin & Jennifer M. Raymond & Stella M. Rouse & Abigael Schirmann \\
\hline Enrique Peruzzotti & Alejandro Razzotti & Lance Roxas & Edella C. Schlager \\
\hline Rosalind P. Petchesky & Sam W.P. Rea, Jr. & Marya Rozanova & Hans Peter Schmitz \\
\hline C. Scott Peters & Daniel J. Reagan & Tom Rozinski & Mark Schneider \\
\hline German Petersen & Leigh-Anne Regenold & Stephen L. Rozman & Marcy Schnitzer \\
\hline V. Spike Peterson & Gary M. Reich & Raul L. Rubio & Andrew J. Schott \\
\hline M.J. Peterson & Arthur J. Reiger & Marva L. Rudolph & Sanford F. Schram \\
\hline Mihail Plamenov Petkov & Mary Beth Reissen & Henry Ruempler & John M. Schroder \\
\hline Mary E. Pettenger & Paul Rejai & Wilma Rule & Philip A. Schrodt \\
\hline Sarah L. Pettijohn & Estanislao Rengifo & Katherine Runge & Livia Isabella Schubiger \\
\hline Edward J. Pfeiffer & Joachim Karl Rennstich & Benjamin Joseph Rusek & Mark D. Schwartz \\
\hline Ngoc Phan & Veronica Reyna & James Taylor Rushing & Brooke Michele Sciotti \\
\hline Justin Phillips & Philip Reynolds & Timothy J. Ryan & Elsie L. Scott \\
\hline Jason Pierceson & Janna King Rezaee & Cynthia K. Ryan & Stephen Scroggs \\
\hline Brian Kristopher Pinaire & Kimberly J. Rice & John Ryan & Lyle A. Scruggs \\
\hline Jessica Piombo & Bradley M. Richardson & Alan Ryan & Evgeny Sedashov \\
\hline Jennifer Pitts & Peter F. Riehm & Rodrigo Saavedra & Steven Seidman \\
\hline Elizabeth Plantan & Neal Riemer & Jennifer Schenk Sacco & Jonathan Serody \\
\hline David J. Plazek & Mary C. Riggall & Gabriella Sacramone-Lutz & Deniz Senol Sert \\
\hline Raymond Polin & Shelley Rigger & Lawrence Saez & Dharma Bahadur Shakya \\
\hline Marc S. Polizzi & Meg E. Rithmire & Sebastian M. Saiegh & Raeann Shane \\
\hline Andrew J. Polsky & Joan Dunne Rittenhouse & Debra J. Salazar & Zijie Shao \\
\hline Laura Polverari & Emily Hencken Ritter & Idean Salehyan & Susan Heilbrunn Shapiro \\
\hline Heather N. Pool & Tesalia Rizzo & Dalal Bajes Salem & Michael J. Shapiro \\
\hline Daniel R. Popkey & Tracie Breshun Roberson & William W. Sales, Jr. & Kam Shapiro \\
\hline Gregory Portillo & Kenneth M. Roberts & Arthur H Saltz & L. Earl Shaw, Jr. \\
\hline Edward B. Portis & Barbara Smith Robertson & Raul Alberto Sanchez & Sarah Shea \\
\hline Brian L. Porto & Ryan Robertson & Urribarri & Susan J. Shearon \\
\hline Robert Postic & Donald W. Robinson & Joe H. Sanchez, Jr. & Ryan M. Sheely \\
\hline Amy R. Poteete & Michael S. Rocca & Lynn M. Sanders & Annelle Rodriguez Sheline \\
\hline Keith A Preble & Javier M. Rodriguez & Arthur Sanders & Edward Sherrill \\
\hline Daniel L. Premo & Raul Rodriguez & Elizabeth Sanders & Shawn Shieh \\
\hline Robert Press & Juan Carlos Rodriguez- & Ellis Sandoz & Masaki Shigeta \\
\hline Melanye Tarea Price & Cordero & Juliana Restrepo Sanin & Harold B. Shill \\
\hline Reagan Pritchett & Philip Roessler & Jack Santucci & Mallory Lauren Shingle \\
\hline Colin Provost & Melvin Lee Rogers & Yusuf Sarfati & Miles H. Shinohara \\
\hline Nattamon Punbhochar & Ingo Rohlfing & Ashutosh Sarker & Charles R. Shipan \\
\hline Kevin F. Quigley & Jeanette Rachelle Rohrhoff & Rodolfo Sarsfield & David A. Shirk \\
\hline John James Quinn & George K. Romoser & Patricia Saulsbery & Boris Shor \\
\hline Dennis P. Quinn & Elizabeth Rooney & Caroline Oldham Saunders & Allyson Shortle \\
\hline David L. Rabin & Laura Roost & Jill E. Savage & Michael Dale Siciliano \\
\hline Bahram Rajaee & Harlan Rosacker & Michael Saward & Edward I. Sidlow \\
\hline Karthick Ramakrishnan & Maria Rosales & Laura J. Scalia & Eleonora Siliprandi \\
\hline Rosemary Ramsey & Justin Rose & Nicole A. Scarborough & Sara Lynn Simeunovic \\
\hline Harry H. Ransom & Peter Rosegg & Frederic C. Schaffer & Evelyn M. Simien \\
\hline
\end{tabular}




\section{Eszter Simon}

Nicole Simonelli

Prerna Singh

James Allen Sink

Althea Rani Sircar

Carmen Joseph Sirianni

Laura Sjoberg

William J. Skane

Susan Navarro Smelcer

Susan Navarro Smelcer

Travis D. Smith

Gary Duane Smith

Daniel E. Smith

Lahra Smith

J.H. Snider

Richard Sobel

David C. Sobelsohn

Femi Razaq Sobowale

Patricia J. Sohn

Sovathana Sokhom

Dorothy J. Solinger

Daniel Solomon

Rosemary Glass Spalding

Katherine Spring

Amber D. Spry

Eoghan Stafford

William D. Stanley

Katina R. Stapleton

Marek D. Steedman

Wayne P. Steger

Rachel M. Stein

Peter J. Steinberger

Maureen S. Steinbruner

Zachary Steinert-Threlkeld

Carolyn Steinle

Sven Steinmo

Mark C. Stephan

Alonzo T. Stephens

Lafleur Stephens

Linda S. Stevenson

Kendra B. Stewart

John G. Stewart

Anna Stilz

Michael Jon Stoil

Donald E. Stokes

Timothy Stoldt

Adam P. Stone

Connie S. Stoner
James R. Stoner, Jr.

Simon A. Stow

Patricia Strach

J. Cherie Strachan

Matt Streb

Anton Strezhnev

Alexander Stroh

Tracy B. Strong

S.I. Strong

Mansoor Suhail

Elizabeth Suhay

Vickie B. Sullivan

Mary Summers

Elizabeth H Super

Glen Sussman

Yusuke Suzumura

Kate Svyatets

Robert H. Swansbrough

Jennie Sweet-Cushman

David Swickard

Ora Beach Szekely

Michael Tabuwe-Aletum

Barry L. Tadlock

William A. Taggart

Susette M. Talarico

Caitlin Talmadge

Jeremy Tamsett

Yuka Tanabe

Yasumasa Tanaka

Haofeng Edward Tang

Holley E. Tankersley

Aron G. Tannenbaum

Christina H. Tarnopolsky

Richard Tashjian

Trevor McMorris Tate

Steven C. Tauber

Scott D. Taylor

Sharon Romayne Taylor

Michelle M. Taylor-Robinson

Jeremy M. Teigen

James R. Terrell, Jr.

Brandon M. Terry

Andrew Thangasamy

Queer J. (Jerry) Thomas

Martha Thompson

Steven Thompson

Danielle Thomsen

Col. John Paul Thornton
John T. Tierney

Seth P. Tillman

Barbara Tobias

Joanna Bache Tobin

Lars Toender

Constantine Trachanas

Andrea V. Traynham

Marc A. Triebwasser

Stacey Triplett

Francisco Jose Troconis

Betty W. Trotter

David L. Trowbridge (Jones)

Chia-hung Tsai

N. Clyde Tucker

Tamelyn Tucker-Worgs

Charles B. Turpin

Syed N. Uddin

Adam Sultan Uddin

William P. Umphres

Mark Ungar

Milada Anna Vachudova

Mona Vakilifathi

Ines Valdez

Henry Valen

Angelo Valente

Nicholas A. Valentino

Arturo A. Valenzuela

Brandon Valeriano

Paul A. Vander Myde

Rachel VanSickle-Ward

Hovhannes R. Vardanyan

Helena Varela

Ivani Vassoler-Froelich

Jacqueline Vaughn

Mayra Velez-Serrano

Ali Velshi

Charles R. Venator-Santiago

Camila Vergara

Angel L. Viera-Tirado

Jose D. Villalobos

Peeranut Visetsuth

Jeffrey J. Volle

James Raymond Vreeland

Kevin Charles Wack

Richard Waghorne

Michael W. Wagner

Joel M. Wagoner

Lo Wing Wah
Paul J. Wahlbeck

Thomas C. Walker

Diane E. Wall

Marc A. Wallace

Teresa T Walsh

Eric Waltenburg

Frederick Carl Walton

Yiwei Wang

Ann Ward

Richard A. Ware

Jean Shumway Warner

Retha W. Warnicke

Anne L. Washington

Charlotte B Watson

Nadja Waxenegger

Marvin G. Weinbaum

Matthew Weinstein

Erika S. Weinthal

Laura C. Weir

Margaret Weir

Akiva Weiss

Danny G. Wells

Russell Andrew West

Gerald T. West

Edward M. Wheat

William Whipple

Beth Elise Whitaker

Julie A. White

Jacqueline A. White

Jean B. White

John Kenneth White

Gregory W. White

Roger W. White

MisChele White

Joseph White

Christopher M. Whitt

Michelle Marie Wietbrock

Nelson Wikstrom

Craig Steven Wilder

Harold L. Wilensky

Susan L. Wiley

Linda Faye Williams

Stewart Hall Williams

Mitchell R. Williams

Leonard Williams

Kay Williams

Thad Williamson

Eliza J. Willis 
Ann R. Willner

Tiffany J. Willoughby-Herard

David C. Wilson

Bruce M. Wilson

Rick Wilson

Pamela Winston

John F. Witte

Jill Wittrock

Jacob P. Wobig

Albert B. Wolf

Deborah Wolf

Eugene Victor Wolfenstein

Scott Wolford

Yvonne Wollenberg

Janet L. Womack

Janelle Wong

James L. Wood

Kristin Lynn Wormley

Fiona M. Wright

Austin Wright

Penyen Wu

Steven T. Wuhs

Clayton Wukich

Jenny Wustenberg

Jinrui Xi

Christina Xydias

Blake Hunter Yagman

Kenneth S. Yalowitz

Masahiro Yamada

Ryusaku Yamada

Kazuo Yamauchi

Priscilla Yamin

Dong-Hoon Yang

Satoko Yasuno

Vickie Ybarra

Adam G. Yoksas

Abigail M. York

Jeffery Allen Yothers, Jr.

Candace C. Young

Iris Marion Young

Jason Scott Young

Mengqi Yuan

Sam Zacher

Michele Zebich-Knos

Eric Zeemering

Mariah Zeisberg

Diana D. Zentay

Chris Zepeda-Millan
Yi Zhao

Zhenqing Zheng

Brandon C. Zicha

Thomas Zittel

Natascha M.E. ZowisloGrünewald

Christina Isabel Zuber

Susanne Zwingel

Department of Health \& Human Services

Ethenomusicologies Work Operas

Lyceum Books, Inc.

University of Massachusetts

Press 